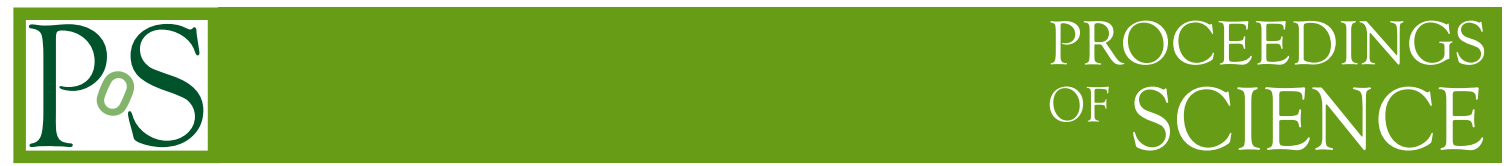

\title{
New proposal for low-emittance muon collider
}

\author{
M. Antonelli, O. R. Blanco-Garcia*, M. Boscolo, S. Guiducci, M. Ricci, M. Rotondo
} INFN Laboratori Nazionali di Frascati, Frascati, Italy
A. Lorenzon, D. Lucchesi, S. Rossini, S. Vanini, M. Zanetti
Università di Padova and INFN Sezione di Padova, Padova, Italy
G. Ballerini, C. Brizzolari, V. Mascagna, M. Prest, M. Soldani
Università degli Studi dell'Insubria e INFN Sezione di Milano Bicocca, Italy

\section{A. Bertolin, F. Gonella, S. Ventura}

INFN Sezione di Padova, Padova, Italy

\section{F. Anulli, F. Collamati, F. lacoangeli ${ }^{\dagger}$}

INFN Sezione di Roma, Roma, Italy

\section{Bandiera ${ }^{\dagger}$}

INFN Sezione di Ferrara, Ferrara, Italy

G. Cavoto ${ }^{\dagger}$

Università di Roma La Sapienza and INFN Sezione di Roma, Roma, Italy

\section{R. Di Nardo}

CERN, Geneva, Switzerland

\section{E. Vallazza}

INFN Sezione di Trieste, Trieste, Italy

\begin{abstract}
These proceedings introduce a new proposal for a low-emittance muon collider. A low-emittance muon beam is obtained from the process $\mathrm{e}^{+} \mathrm{e}^{-} \rightarrow \mu^{+} \mu^{-}$with positrons at the threshold energy of $45 \mathrm{GeV}$. A first test beam to validate the low-emittance muon production concept will be presented.
\end{abstract}

Sixth Annual Conference on Large Hadron Collider Physics (LHCP2018)

4-9 June 2018

Bologna, Italy

\footnotetext{
* Speaker.

${ }^{\dagger}$ the author acknowledge the support of CRYSBEAM project GA n. 615089.
} 


\section{Introduction}

The future beyond the LHC discoveries opens up to a next generation of facilities exploring the energy frontier in particle physics. In such perspective, a muon collider at the multi-TeV offers great potential if it is shown feasible [1].

Studies of neutrino factories and muon colliders have been on-going since the 1990's in the U.S.A. by the Muon Accelerator Program (MAP) [2], where muons come from the decay of pions obtained from the collision of protons with a fixed target [3]. Surface muons, the muons that decay from pions on the material surface, are collected to form an intense muon beam with a large beam emittance. Therefore, muon cooling is the next step in the MAP accelerator chain conceptual design where emittance in the longitudinal and transverse planes is reduced to achieve higher luminosity. The transverse emittance cooling concept has been tested by the MICE [4] experiment at the Rutherford Appleton Laboratory in England, finishing its program with positive results which are now public. The longitudinal cooling has not been put to test yet.

The Low EMittance Muon Accelerator (LEMMA) project [5] is studying the possibility of a muon collider, with performance similar to that of MAP, where muons are produced already with small emittance. This novel idea might change the future of muon colliders as it might not need cooling. Muons are produced from annihilation of a high intensity positron beam with electrons in a target [6]. In order to produce muons at threshold, a $45 \mathrm{GeV}$ positron beam collides with a fixed target.

There are several challenges for the low emittance muon source of LEMMA. First, the muon production comes from an small cross section, 0.1 to $1 \mu$ barn, channel: $\mathrm{e}^{+} \mathrm{e}^{-} \rightarrow \mu^{+} \mu^{-}$. To generate a large number of muons this requires a high positron rate. LEMMA is considering the recirculation of a positron beam in a $6.3 \mathrm{~km}$ long ring, see Fig. 1, where the positron bunches interact every turn with the target. In addition, the muons produced must be accumulated, therefore, two muon accumulator rings are put in place.

A second challenge is the thermo-mechanical stress in the target. Several target options are currently under consideration, in particular a solid beryllium target seems to balance muon production rate and positron beam degradation.

Third, contributions to beam emittance growth should be minimized from the source up to the collision point. There are several contributions to the beam emittance growth, but initial studies of the positron ring dynamics show that matching the beam with the target phenomenology leads to a beam emittance growth under control [5].

LEMMA has started to move towards experiments that validate the low emittance muon production concept. In the following we describe the 2017 LEMMA test beam that occurred during the 7 days between July 26th and August 2th at the H4 line of the CERN North Area.

\section{Experimental setup}

The experimental setup as reconstructed in Geant4 is shown in Fig. 2. The coordinate system used has the $z$ axis pointing along the incoming positron beam direction, the $y$ axis pointing upwards and the $x$ axis completing a right handed coordinate system. Two silicon tracking devices, $2 \times 2$ $\mathrm{cm}^{2}$, labeled T1 and T2 [7], were placed upstream of the $6 \mathrm{~cm}$ long beryllium target. These are 


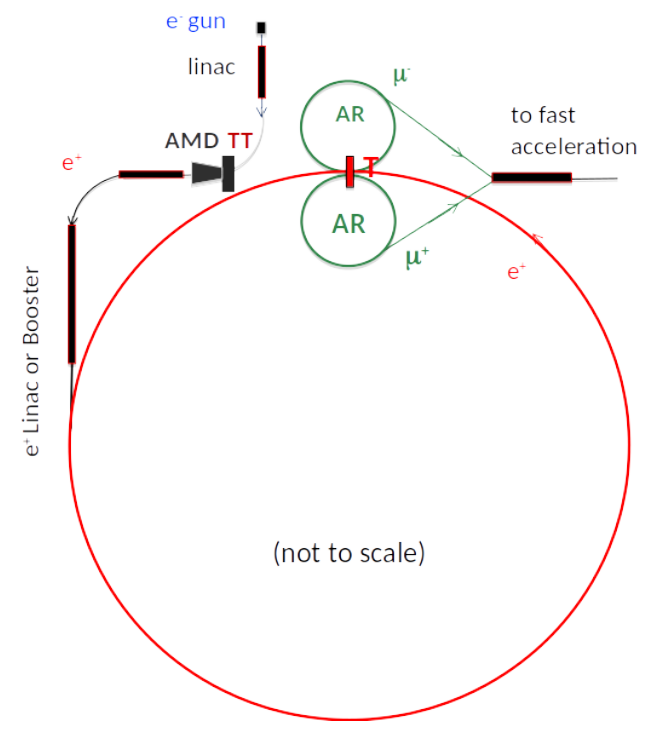

Figure 1: LEMMA accelerator concept. The beam in the Positron Ring, in red, interacts with the Target (T) every turn. Muons are accumulated in the accumulator rings (AR) during less than one lifetime and then extrated for further acceleration.

used to measure the direction of the incoming positron(s). Downstream of the target but upstream of the magnetic field region, two silicon devices, T3, $2 \times 2 \mathrm{~cm}^{2}$, and $\mathrm{C} 1$ [8], $10 \times 10 \mathrm{~cm}^{2}$, were measuring the beam positrons surviving the passage trough the target as well as any additional charged particle produced by the incoming beam. A magnet was used in order to produce the deflection corresponding to a $1.26 \mathrm{~T}$ magnetic field directed along the $y$ axis acting on a $2 \mathrm{~m}$ distance. This value was used to ensure a good separation between $45 \mathrm{GeV}$ positron tracks and positive tracks in the $[18,26] \mathrm{GeV}$ momentum range. Negative tracks were deflected towards the silicon devices $\mathrm{C} 3$ and $\mathrm{C} 5$, both $10 \times 10 \mathrm{~cm}^{2}$, and the negative $x$ side of a large, about $2 \mathrm{~m}$ wide and $1 \mathrm{~m}$ height, drift tubes device, a (spare) CMS muon chamber. C3 and C5 where placed in order to have good acceptance in the momentum range $[18,26] \mathrm{GeV}$. Positive tracks in the same momentum range were recorded in the silicon devices $\mathrm{C} 2$ and $\mathrm{C} 4{ }^{1}$, both $10 \times 10 \mathrm{~cm}^{2}$. Positrons were expected to deposit most of their energy in the Ecal, a lead glass calorimeter. Any eventual leakage was absorbed by the iron shielding placed downstream. Hence only $\mu^{+}$tracks were expected to be recorded in the positive $x$ side of the muon chamber. Photons emerging from the target were absorbed in a PbWO4 calorimeter, the $\gamma$ cal. Finally a Cherenkov detector is placed downstream of the negative $x$ side of the muon chamber to differentiate between electron and muon tracks, with acceptance in the $[18,26] \mathrm{GeV}$ momentum range. A trigger was provided using a triple coincidence between scintillator pads located upstream of T1 and downstream of C4 and C5. Silicon detectors were providing hits along the $x$ and $y$ axis. The pitch between readout strips was in the range between 5 and $25 \mu \mathrm{m}$, depending on the size of the silicon detector, the smaller detectors having also the smaller pitch. The muon chamber was providing 8 hits along the $x$ axis, bending axis, with

\footnotetext{
${ }^{1}$ The authors acknowledge the hardware support from the colleagues of INFN Sezione di Bari, essential for the setup and operations of all the "large" silicon devices.
} 
a $150 \mu \mathrm{m}$ expected resolution, and 4 along the $y$ axis, with a $200 \mu \mathrm{m}$ expected resolution.

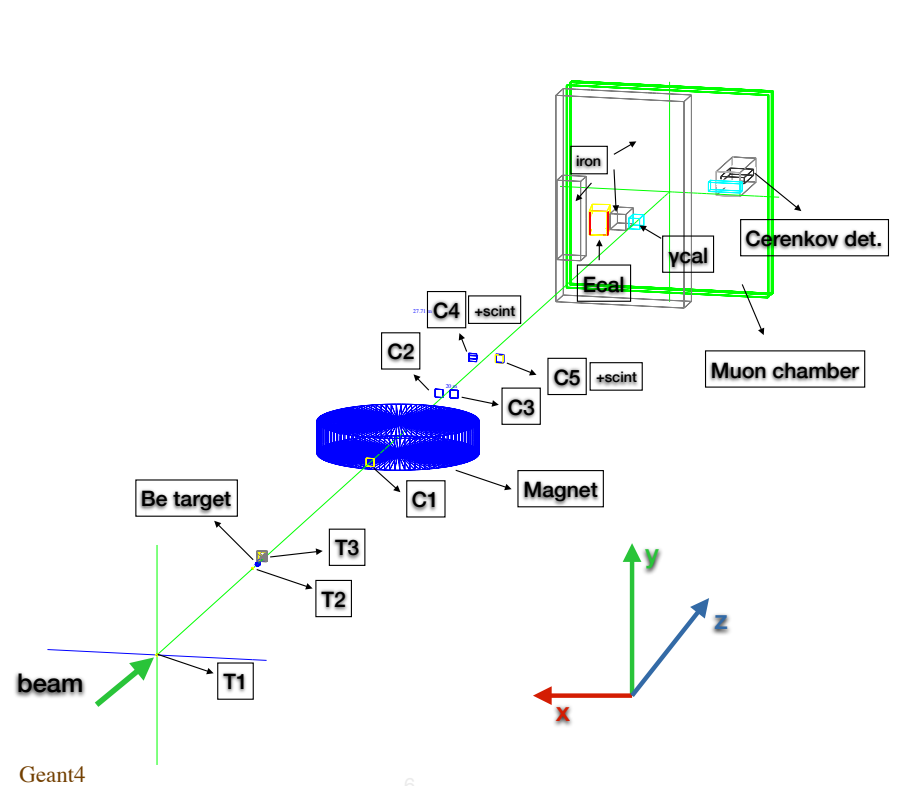

Figure 2: Test beam experimental setup.

\section{Analysis strategy}

Several unexpected hardware difficulties occurred during the 7 days test beam that occurred between July 26th and August 2th 2017. As a consequence useful calibrations and physics runs could only be recorded starting from August 1st. These include:

- a calibration run, without target, with positrons at $22 \mathrm{GeV}$;

- two positrons calibration runs, without target, at 18 and $22 \mathrm{GeV}$, taken with a reversed magnetic field to mimic negatively charged tracks of the same energy;

- several "physics" runs, with the target in and a $45 \mathrm{GeV}$ impinging positron beam.

The two calibrations runs with reversed magnetic field have been used for overall detector alignment and momentum calibration. The other calibration run was used to assess that the probability for a position to give a track in the muon chamber is null: no reconstructed track was found within the available statistic of about 4000 incoming positron triggers.

The signal of interest corresponds to the process:

$$
e^{+} e^{-} \rightarrow \mu^{+} \mu^{-}
$$

hence two tracks reconstructed in the muon chamber were looked for. Both tracks where first propagated backward to the silicon detectors $\mathrm{C} 2-\mathrm{C} 4$ and $\mathrm{C} 3-\mathrm{C} 5$ with a linear extrapolation model. Using an estimate of the tracks momentum obtained from the position recorded in the muon chamber, the two tracks are additionally propagated to $\mathrm{C} 1$ using a circular track model. Finally tracks 
are connected to hits in T3 by linear extrapolation. At this stage all hits are fitted again to obtain the best estimate of the track momentum and angle in the bending plane, the $x-z$ plane. The candidate events contain one $\mu^{+}$and one $\mu^{-}$track for which the momentum fit converged.

\section{Results}

With the procedure outlined in the previous section $27 \mu^{+} \mu^{-}$candidate events were obtained. The momentum and angular distributions at $\mathrm{C} 1$ are shown separately for positive and negative selected tracks in Fig. 3 and compared with the corresponding distributions obtained from a sample of simulated $\mu^{+} \mu^{-}$events obtained with the BabaYaga[9] event generator and tracked with Geant4[10]. The simulated events have been processed with the same algorithms and selections as the real data. A fair agreement between data and simulation is observed.
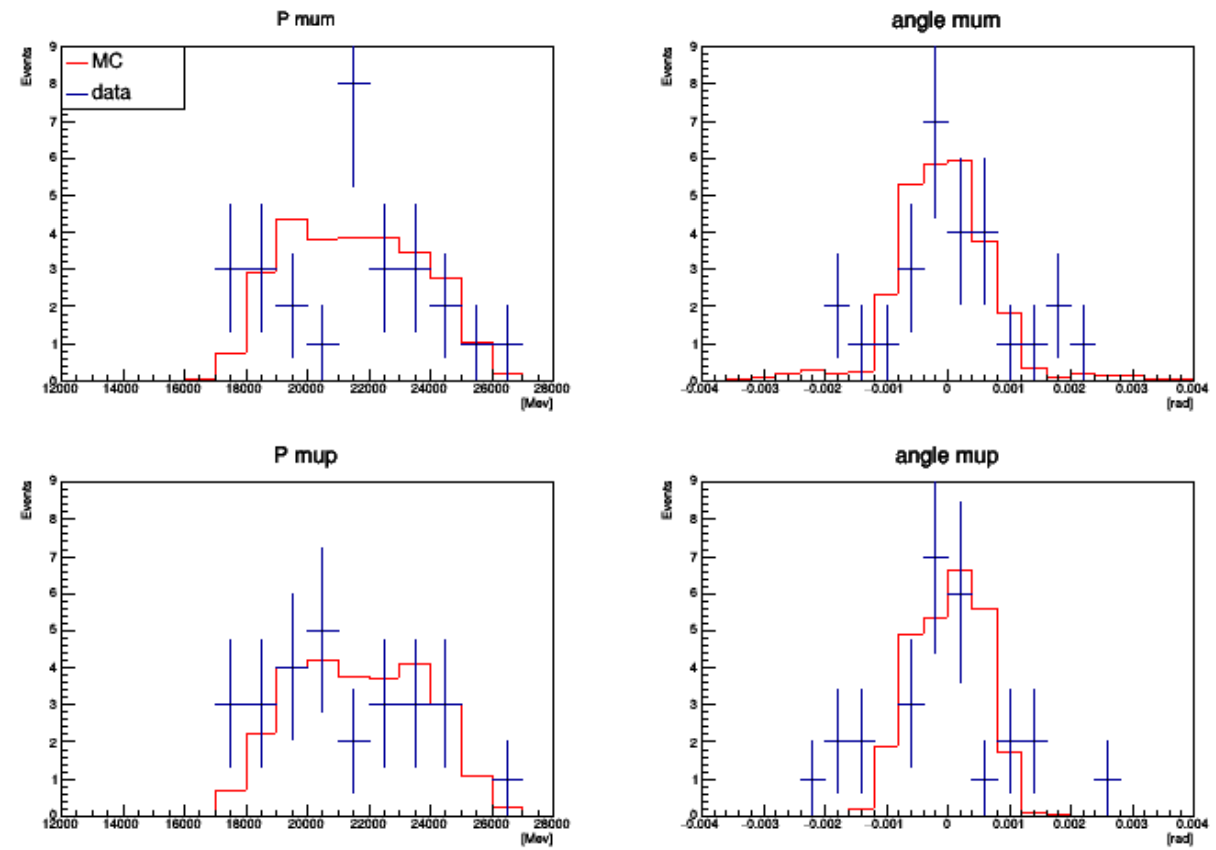

Figure 3: Reconstructed momentum, left, and angle in the bending plane, right, for negatively, upper, and positively, lower, charged tracks both reaching the muon chamber in data and MC.

Two observables more sensitive to the $e^{+} e^{-} \rightarrow \mu^{+} \mu^{-}$underlying physics have also been considered. The first one is the energy sum of the final state muon tracks which is clearly expected to peak at the energy of the incoming beam, $45 \mathrm{GeV}$. The distribution observed in data is shown by the left plot in Fig. 4 and compared to the corresponding simulation result, obtained taking into account the energy resolution measured in the calibration runs, $\sigma_{p} / p=3.6 \%$ at $18 \mathrm{GeV}$ with a mild momentum dependence. The second one is the invariant mass of the final state muon pair: data and simulation are compared in the right plot of Fig. 4. For both observables, within the limited statistic available, a reasonable agreement between data and simulation is found.

The number of signal candidates identified is clearly not sufficient for a measurement of the emittance. Hence a new test beam, with an improved hardware setup, was performed in Summer 

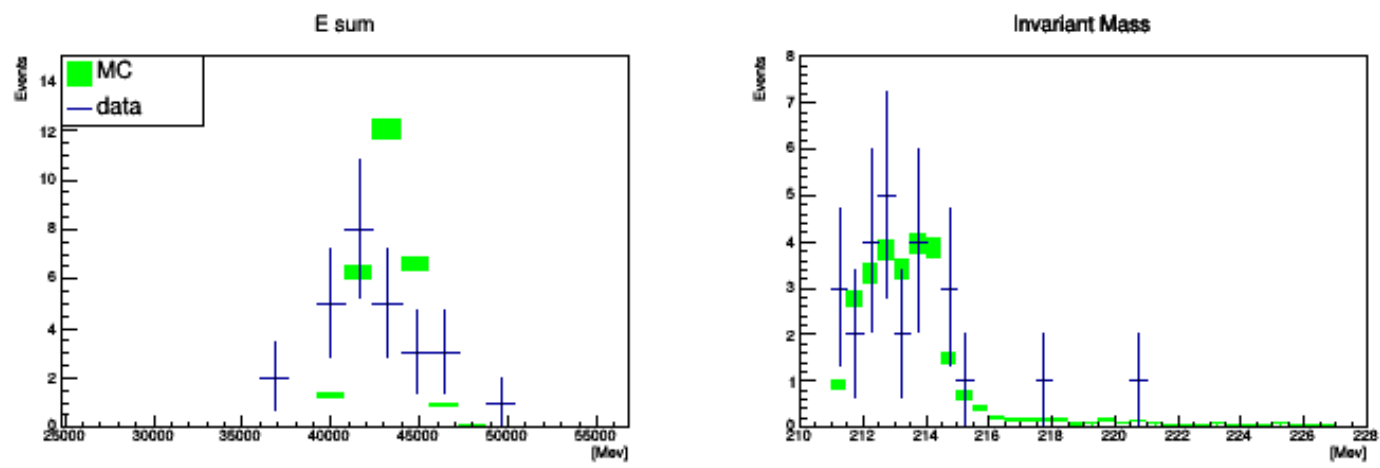

Figure 4: Energy sum, left, and invariant mass, right, of the $\mu^{+} \mu^{-}$tracks in data and MC.

2018. At the time these proceedings are beeing submitted the analysis of the 2018 test beam data has just begun.

\section{References}

[1] F. Zimmermann. Future Colliders for Particle Physics - "Big and Small”. Arxiv:1801.03170. Submitted to the proceedings of the Third European Advanced Accelerator Concepts Workshop "EAAC2017", La Biodola, 24-30 September 2017. To be published in NIMA Proceedings.

[2] R. D. Ryne et. al. Design Concept for Muon-Based Accelerators, WEPWA057, in proceedings of IPAC 2015, Richmond, VA, U.S.A.

[3] F. Berg. Development of a Next-generation High-Intensity Muon Beam at the Paul Scherrer, LNF Mini-Workshop Series: Muon production and beam interceptors. April-19, 2018. LNF/INFN, Frascati, Italy

[4] T. A. Mohayai et. al. First Demonstration of Ionization Cooling in MICE, FRXGBE3, in proceedings of IPAC 2018, Vancouver, BC, Canada

[5] M. Boscolo. et. al. Low Emittance Muon Accelerator Studies with production from positrons on target. Physics. Rev. Accel. Beams, 21-6, 2018

[6] M. Antonelli. et. al. Novel Proposal for a Low Emittance Muon beam using positron beam on target. Nucl. Instr. Meth. A 807, 101-107 (2015)

[7] D. Lietti et al., A microstrip silicon telescope for high performance particle tracking. Nucl. Instr. Meth. A 729, 527-536 (2013)

[8] G. Barbiellini et al., The AGILE silicon tracker: testbeam results of the prototype silicon detector. Nucl. Instr. Meth. A 490, 146-158 (2002)

[9] G. Balossini et al., Matching perturbative and Parton Shower corrections to Bhabha process at flavour factories, Nucl. Phys. B758, 227-253 (2006)

[10] Geant4 collaboration, J. Allison et al., Geant4 developments and applications, IEEE Trans. Nucl. Sci. 53 (2006) 270

Geant4 collaboration, S. Agostinelli et al., Geant4: A simulation toolkit, Nucl. Instrum. Meth. A506 (2003) 250 